\title{
Multiple Spectral Splits of Supernova Neutrinos
}

\author{
Basudeb Dasgupta, ${ }^{1}$ Amol Dighe, ${ }^{2}$ Georg G. Raffelt, ${ }^{1}$ and Alexei Yu. Smirnov ${ }^{3,4}$ \\ ${ }^{1}$ Max-Planck-Institut für Physik (Werner-Heisenberg-Institut), Föhringer Ring 6, 80805 München, Germany \\ ${ }^{2}$ Tata Institute of Fundamental Research, Homi Bhabha Road, Mumbai 400005, India \\ ${ }^{3}$ Abdus Salam International Centre for Theoretical Physics, Strada Costiera 11, 34014 Trieste, Italy \\ ${ }^{4}$ Institute for Nuclear Research, Russian Academy of Sciences, 117312 Moscow, Russia
}

(Dated: 19 April 2009, revised 17 June 2009)

\begin{abstract}
Collective oscillations of supernova neutrinos swap the spectra $f_{\nu_{e}}(E)$ and $f_{\bar{\nu}_{e}}(E)$ with those of another flavor in certain energy intervals bounded by sharp spectral splits. This phenomenon is far more general than previously appreciated: typically one finds one or more swaps and accompanying splits in the $\nu$ and $\bar{\nu}$ channels for both inverted and normal neutrino mass hierarchies. Depending on an instability condition, swaps develop around spectral crossings (energies where $f_{\nu_{e}}=f_{\nu_{x}}$, $f_{\bar{\nu}_{e}}=f_{\bar{\nu}_{x}}$ as well as $E \rightarrow \infty$ where all fluxes vanish), and the widths of swaps are determined by the spectra and fluxes. Wash-out by multi-angle decoherence varies across the spectrum and splits can survive as sharp spectral features.
\end{abstract}

PACS numbers: 14.60.Pq, 97.60.Bw

Introduction.-The neutrino flux from a core-collapse supernova $(\mathrm{SN})$ is a powerful probe of particle physics and astrophysics [1]. SN neutrinos interact not only with the stellar medium, producing the Mikheyev-SmirnovWolfenstein (MSW) flavor conversion, but also with other neutrinos and antineutrinos. The latter interactions modify the flavor evolution in a non-linear fashion and give rise to collective forms of oscillation $2,2,3,4,5,6,6,7$, a subject of intense recent investigation $[8,99,10,11,12$, $13,14,15,16,17,18,19,20,21,222,23,24,25,26,27$, $28,29,30,31$.

The most important observational consequence of the collective effects is an exchange of the $\nu_{e}\left(\bar{\nu}_{e}\right)$ spectrum with the $\nu_{x}\left(\bar{\nu}_{x}\right)$ spectrum in certain energy intervals. We call such a flavor exchange a "swap", whereas "splits" are sharp boundary features at the edges of each swap interval. Spectral splits may become observable in the high-statistics neutrino signal from the next galactic SN, leading to valuable clues about the underlying physics [19, 23, 26].

The well-understood "classic swap" covers the entire $\bar{\nu}$ spectrum and that of $\nu$ above an energy fixed by the approximate conservation of the $\nu_{e}$ deleptonization flux [16, 17, 18]. In this paper we show that spectral swaps and concomitant splits are more ubiquitous than has been appreciated in the past. One example is the puzzling low-energy split in the $\bar{\nu}$ spectrum that was noted for the inverted neutrino mass hierarchy [20, 21]. However, with flavor spectra typical for SN neutrinos one should expect multiple splits in either hierarchy.

We focus on neutrino-neutrino interactions alone and study two-flavor oscillations driven by the atmospheric mass difference and 1-3 mixing. As has been established before [14], the usual matter effect in the region of collective oscillations (up to a few $100 \mathrm{~km}$ ) can be accounted for by choosing a small (matter suppressed) effective mixing angle which we take to be $\theta_{\text {eff }}=10^{-5}$. MSW conver-

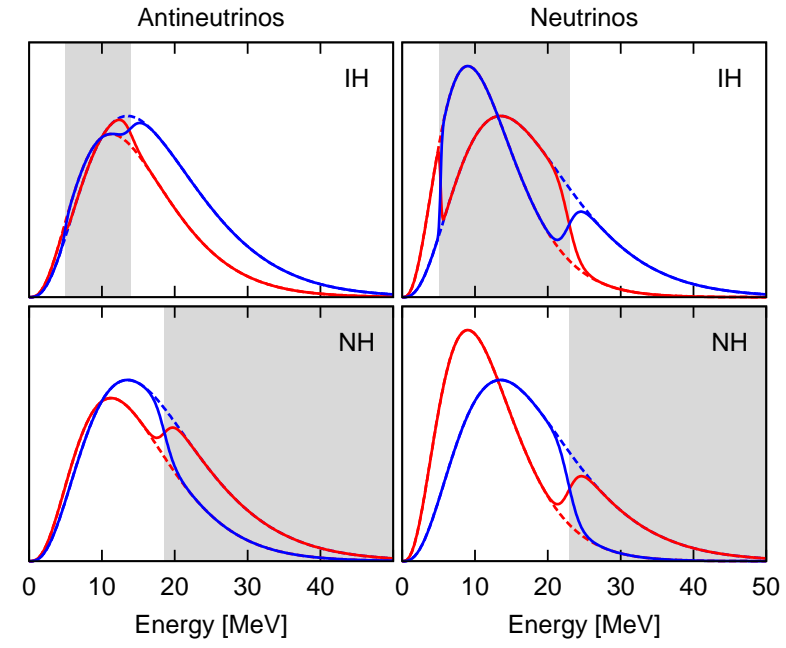

FIG. 1: SN neutrino spectra before (dashed lines) and after (solid lines) collective oscillations, but before possible MSW conversions. The panels are for $\nu$ and $\bar{\nu}$, each time for IH and NH. Red lines $e$-flavor, blue $x$-flavor. Shaded regions mark swap intervals.

sions occur typically at larger distances. Their effects then factorize and can be included separately 24].

Spectral crossings and spectral swaps.-Consider first the SN cooling phase where plausible choices are 32. $F_{\nu_{e}}: F_{\bar{\nu}_{e}}: F_{\nu_{x}}=0.85: 0.75: 1.00$ for the neutrino fluxes, $\bar{E}_{\nu_{e}}=12, \bar{E}_{\bar{\nu}_{e}}=15$ and $\bar{E}_{\nu_{x}}=\bar{E}_{\bar{\nu}_{x}}=18 \mathrm{MeV}$ for the average energies, and $f_{\nu}(E) \propto E^{3} e^{-4 E / \bar{E}}$ for the spectral shape. Based on the single-angle approximation for neutrino propagation [11, 14, 20, 28], Fig. 1]shows the flavor spectra before and after collective oscillations. For the inverted mass hierarchy (IH) we find a swap for both $\nu$ and $\bar{\nu}$ and thus a total of four splits. For the normal hierarchy $(\mathrm{NH})$ the swaps extend to infinite $E$, providing one split in the $\nu$ and $\bar{\nu}$ spectrum each.

Flavor oscillations leave $f_{\nu_{e}}(E)+f_{\nu_{x}}(E)$ unchanged, 


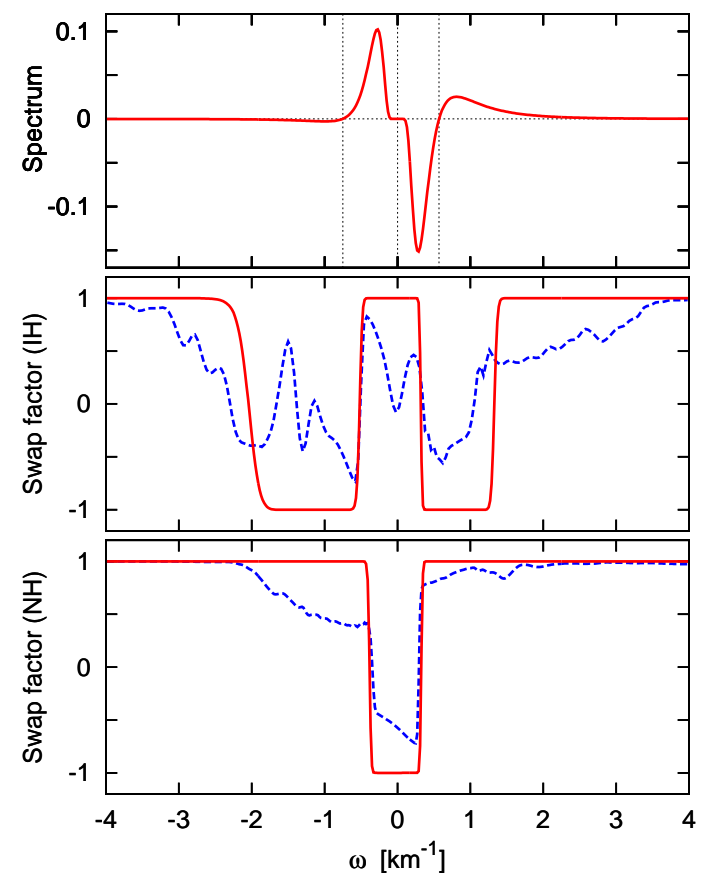

FIG. 2: Initial spectrum $g_{\omega}$ defined in Eq. (1) for the coolingphase example of Fig. 1. Swap factor after collective oscillations for IH and NH as indicated. Single-angle (solid) and multi-angle (dashed). For animations see Ref. [33].

so $\Delta f_{\nu}(E) \equiv f_{\nu_{e}}(E)-f_{\nu_{x}}(E)$, and similarly for $\bar{\nu}$, contains all relevant information. It proves crucial for understanding the multisplit phenomenon (i) to use $-\Delta f_{\bar{\nu}}(E)$ for $\bar{\nu}$ (the "flavor isospin" construction [1]), and (ii) to merge the $\nu$ and $\bar{\nu}$ spectra to a single continuum in terms of the variable $\omega \equiv \pm\left|\Delta m^{2} / 2 E\right|$, the vacuum oscillation frequency, that is positive for $\nu$ and negative for $\bar{\nu}$. Here $\Delta m^{2} \equiv m_{2}^{2}-m_{1}^{2}$ with $\nu_{1} \approx \nu_{e}$ and $\nu_{2} \approx \nu_{x}$, so $\Delta m^{2}>0$ stands for $\mathrm{NH}$ and $\Delta m^{2}<0$ for IH. With $E_{\omega}=\left|\Delta m^{2} / 2 \omega\right|$ we thus define our spectrum as

$$
g_{\omega} \equiv \frac{\left|\Delta m^{2}\right|}{2 \omega^{2}} \times \begin{cases}f_{\nu_{e}}\left(E_{\omega}\right)-f_{\nu_{x}}\left(E_{\omega}\right) & \text { for } \omega>0 \\ f_{\bar{\nu}_{x}}\left(E_{\omega}\right)-f_{\bar{\nu}_{e}}\left(E_{\omega}\right) & \text { for } \omega<0\end{cases}
$$

where the common factor comes from the $E \rightarrow \omega$ transformation. In SN we have $f_{\nu_{x}}(E)=f_{\bar{\nu}_{x}}(E)$ and a net $\nu_{e}$ deleptonization flux, so $\int_{-\infty}^{+\infty} \mathrm{d} \omega g_{\omega}>0$. Notice that $g_{\omega}>0$ means excess $\nu_{e}$ for $\omega>0$ and excess $\bar{\nu}_{x}$ for $\omega<0$.

In Fig. 2 we show $g(\omega)$ corresponding to the example of Fig. [1 The effect of collective oscillations is represented by a "swap factor" $S_{\omega}$, giving the final spectrum as

$$
g_{\omega}^{\text {final }}=S_{\omega} g_{\omega}^{\text {initial }} .
$$

The "spectral crossings" where $g(\omega)=0$ are indicated by vertical dotted lines. In particular, our construction provides a $\nu-\bar{\nu}$ crossing at $\omega=0$ (infinite $E$ ), playing the same role as a "true flavor crossing" where $f_{\nu_{e}}(E)=$ $f_{\nu_{x}}(E)$. The $\nu-\bar{\nu}$ crossing exists even if the source emits only $\nu_{e}$ and $\bar{\nu}_{e}$.

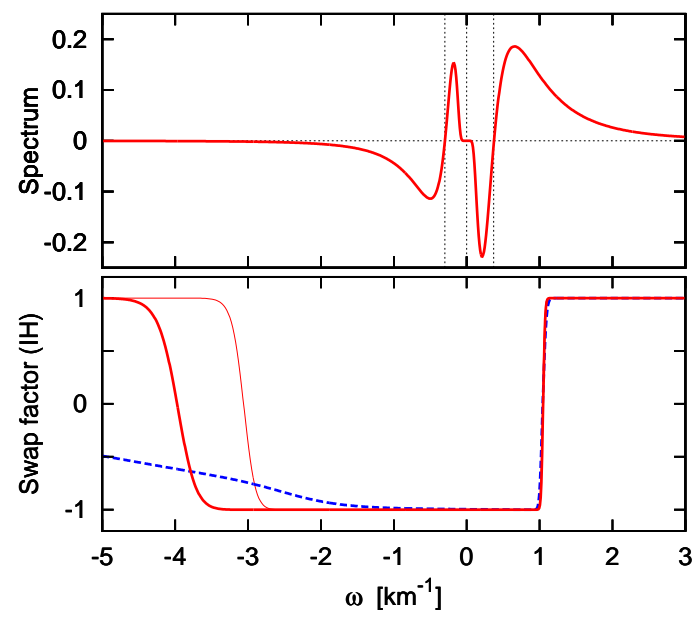

FIG. 3: Same as Fig. 2 for the accretion-phase example of Refs. 20, 21]. Thin solid line: swap factor for $\theta_{\text {eff }}=10^{-10}$ instead of $10^{-5}$. There is no resolved swap for $\mathrm{NH}$.

Figure 2 illustrates a general point: for IH every $g_{\omega}$ crossing with a positive slope is an "unstable crossing", i.e. it induces a swap over a finite $\omega$ range with splits that become sharp in the adiabatic limit. For NH every $g_{\omega}$ crossing with a negative slope is an unstable crossing. (The equations of motion actually reveal that the dynamics for a given spectrum $g_{\omega}$ in IH is equivalent to a spectrum $-g_{\omega}$ in $\mathrm{NH}$, thus there is no fundamental difference between the two hierarchies.)

The usual assumptions about SN neutrinos lead to a triple-crossed spectrum and thus to one or two swaps (two or four splits), depending on the hierarchy. An apparent counter example, representing the SN accretion phase, is $\bar{E}_{\nu_{e}}=10, \bar{E}_{\bar{\nu}_{e}}=15$ and $\bar{E}_{\nu_{x}}=\bar{E}_{\bar{\nu}_{x}}=24 \mathrm{MeV}$ and $F_{\nu_{e}}: F_{\bar{\nu}_{e}}: F_{\nu_{x}}=2.4: 1.6: 1.0[20$, 21]. The corresponding $\omega$ spectrum (Fig. 3) has three crossings, yet only one swap is observed for IH and none for NH. Below we argue that the narrow spacing of the crossings implies that (i) in the adiabatic limit the missing swap is exponentially narrow, and (ii) it is suppressed by adiabaticity violation. Therefore, a narrowly spaced triple crossing can superficially act like a single one.

Single-crossed system.-Many of our general observations are borne out from a generic single-crossed example for IH (Fig. (4). The equations of motion (EOMs) are

$$
\dot{\mathbf{P}}_{\omega}=(\omega \mathbf{B}+\mu \mathbf{P}) \times \mathbf{P}_{\omega},
$$

where $\mathbf{B}$ is a unit vector $(0,0,1)$ in flavor space, $\mathbf{P}_{\omega}$ is the polarization-vector spectrum that covers $\nu$ for $\omega>0$ and $\bar{\nu}$ for $\omega<0$, and $\mathbf{P}=\int_{-\infty}^{+\infty} \mathrm{d} \omega \mathbf{P}_{\omega}$. The coupling $\mu$ depends on $G_{\mathrm{F}}$, the local $\nu$ and $\bar{\nu}$ densities, and the normalization of $g_{\omega}$. Initially $\mathbf{P}_{\omega}=\left(0,0, g_{\omega}\right)$, for a vanishingly small mixing angle. The crossing is unstable for $\mathrm{IH}$ because a swap would decrease the energy $\int d \omega \omega \mathbf{B} \cdot \mathbf{P}_{\omega}$. (For NH, the $\omega \mathbf{B}$ term in Eq. (3) appears with a negative sign, so $\int d \omega \omega \mathbf{B} \cdot \mathbf{P}_{\omega}$ is already minimal. However, 


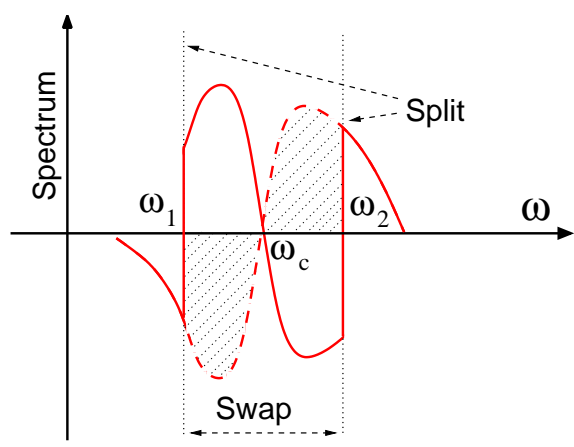

FIG. 4: Basic case of a spectrum producing a swap. The initial spectrum (dashed) has an unstable crossing at $\omega_{c}$, whereas the final spectrum (solid) has splits at $\omega_{1}$ and $\omega_{2}$.

a spectrum $-g_{\omega}$ would have an unstable crossing.) We seek the final spectrum for this schematic case, when $\mu$ slowly decreases from some initial value $\mu_{0}$ to 0 .

The EOMs imply that $\mathbf{B} \cdot \mathbf{P}=\int \mathrm{d} \omega g_{\omega}$ is conserved, so we can "rotate away" a common precession of the entire system with frequency $\mu \mathbf{B} \cdot \mathbf{P}$ in the same way as removing the usual matter term [27]. The EOMs become

$$
\dot{\mathbf{P}}_{\omega}=\left(\omega \mathbf{B}+\mu \mathbf{P}_{\perp}\right) \times \mathbf{P}_{\omega}
$$

where $\mathbf{P}_{\perp}$ is the component of $\mathbf{P}$ transverse to $\mathbf{B}$.

For $\mathbf{B} \cdot \mathbf{P} \neq 0$ there is a critical value $\mu_{\text {sync }}$ such that for $\mu_{0}>\mu_{\text {sync }}$ the system initially performs synchronized oscillations with $\mu \mathbf{P}_{\perp}=0$. As $\mu$ decreases, each $\mathbf{P}_{\omega}$ follows its Hamiltonian $\omega \mathbf{B}+\mu \mathbf{P}_{\perp}$, performing a "pure precession," and all $\mathbf{P}_{\omega}$ with $\omega<\omega_{\text {split }}$ end anti-aligned with $\mathbf{B}$, the others aligned, leading to the classic split fixed by B.P conservation [16, 17, 18]. For B.P $=0$ and $\mu_{0} \rightarrow \infty$, the system evolves like the flavor pendulum [12], ending with the entire spectrum swapped.

A qualitatively different behavior occurs for $\mu_{0}<$ $\mu_{\text {sync }}$, or any finite $\mu_{0}$ if $\mathbf{B} \cdot \mathbf{P}=0$. Now $\mu \mathbf{P}_{\perp}$ performs a fast non-adiabatic motion with large amplitude, causing a collective oscillation of the entire spectrum. This generic behavior is borne out analytically for a spectrum that is antisymmetric in $\omega$ relative to $\omega_{c}$, leading to a "pure nutation" in terms of a variable $\varphi(t)$. Without loss of generality we use $\omega_{\mathrm{c}}=0$ and find 30, 31],

$$
\mathbf{P}_{\omega}=\frac{g_{\omega}}{\sqrt{\omega^{4}-2 \omega^{2} \kappa^{2} c_{\mathrm{m}}+\kappa^{4}}}\left(\begin{array}{c}
-\kappa^{2} s \\
\omega \kappa \sqrt{2\left(c-c_{\mathrm{m}}\right)} \\
\omega^{2}-\kappa^{2} c
\end{array}\right)
$$

where $s \equiv \sin \varphi, c \equiv \cos \varphi, c_{\mathrm{m}} \equiv \cos \varphi_{\max }$, and $\varphi(t)$ obeys $\frac{1}{2} \dot{\varphi}^{2}=\kappa^{2}\left(\cos \varphi-\cos \varphi_{\max }\right)$. Differentiation provides $\ddot{\varphi}=-\kappa^{2} \sin \varphi$, the equation for an anharmonic oscillator. Equation (5) solves the EOM (44) if the nutation amplitude $\varphi_{\max }$ and frequency $\kappa$ fulfill the consistency relation

$$
\int \mathrm{d} \omega \frac{\omega g_{\omega}}{\sqrt{\omega^{4}-2 \omega^{2} \kappa^{2} c_{\mathrm{m}}+\kappa^{4}}}=\frac{1}{\mu} .
$$

For a "symmetric box" where $g(\omega)=1 / \Delta \omega$ for $0<\omega<$ $\frac{1}{2} \Delta \omega$ and $g_{-\omega}=-g_{\omega}$ we find $\kappa_{0}=\Delta \omega / \sqrt{\mathrm{e}^{\Delta \omega / \mu_{0}}-1}$.

The initial state when $\mu=\mu_{0}$ is described by $\varphi=$ $\varphi_{\max }=\pi$, implying $c_{\mathrm{m}}=-1$ and so the amplitude of $\mathbf{P}_{\omega}^{z}$ in Eq. (5) is $\kappa_{0}^{2} /\left(\omega^{2}+\kappa_{0}^{2}\right)$. The modulation of $g_{\omega}$ thus has a resonance with a maximum at $\omega=0$. As $\mu$ decreases adiabatically from $\mu_{0}$ to 0 , the r.h.s. of Eq. (6) becomes singular and so the l.h.s. must become singular as well. This is only possible for $c_{\mathrm{m}} \rightarrow+1$, i.e., the final maximum nutation amplitude is $\varphi_{\max }=0$. At the same time, the frequency $\kappa$ decreases from $\kappa_{0}$ to some non-zero value $\kappa_{\mathrm{s}}$. With $c_{\mathrm{m}}=+1$, Eq. (5) provides $S_{\omega}=\left(\omega^{2}-\kappa_{\mathrm{s}}^{2}\right) /\left|\omega^{2}-\kappa_{\mathrm{s}}^{2}\right|= \pm 1$, and thus there is a swap in the range of frequencies $\omega^{2}<\kappa_{\mathrm{s}}^{2}$. In other words, the final $\kappa$ plays the role of the split frequency $\omega_{\text {split }}= \pm \kappa_{\mathrm{s}}$, which is related to $\kappa_{0}$ through an adiabatic invariant.

Because $\kappa_{0}$ plays the dual role of the approximate swap width and an inverse evolutionary time scale, an exponentially small $\kappa_{0}$ implies that the corresponding swap disappears (i) by not being numerically resolved and (ii) by adiabaticity violation.

A non-zero mixing $\left(0<\theta_{\text {eff }} \ll 1\right)$ is required to trigger the initial motion, but also causes a delay until the system relaxes to the adiabatic solution. Therefore, the effective $\mu_{0}$ relevant for swap formation is reduced and the swap width is smaller for a smaller $\theta_{\text {eff }}$. This subtle effect can become visible when a split falls into a tail of $g_{\omega}$ where the flux is small. A case in point is the left split in Fig. 3 that shifts to the right for a smaller $\theta_{\text {eff }}$ (thin-line), explaining the dependence on the matter profile (that modifies $\theta_{\text {eff }}$ ) noted in Refs. 20, 21]. Similar remarks pertain to the left-most IH split in Fig. 2

Multiple crossings. - For a spectrum with several crossings the evolution is more complicated and depends on various features, notably the separation of the crossings and $\mu_{0}$. For instance, a multi-crossed system can be con-

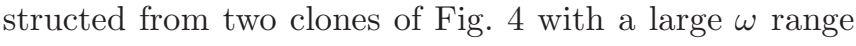
of empty modes between them. If $\mu_{0}$ is small, each individual block shows the behavior discussed above. Even though the entire spectrum feels the same $\mu \mathbf{P}_{\perp}(t)$, only the Fourier components close to a given $\omega$ strongly affect $\mathbf{P}_{\omega}$, so different regions of the spectrum can evolve almost independently of each other. If $\mu_{0}>\mu_{\text {sync }}$ of a single clone, we get the "co-rotating plane solution" and classic split in each clone, once more because distant Fourier components of $\mathbf{P}_{\perp}$ do not affect a given solution. If $\mu_{0}$ is so large that both clones are synchronized with each other, a true adiabatic solution would lead to one single classic split for the entire spectrum, but this solution is unstable. Once $\mu$ has become smaller than the separation between the clones, the classic co-rotation solution for the entire spectrum gives way to two approximately independent solutions, one for each clone.

Most interesting in the SN context is a triple-crossed spectrum. For $\mathrm{NH}$ we have a single unstable crossing flanked by two stable ones, resulting in a single swap 
(Fig. 2) that can be understood, using the appropriate $g_{\omega}$ in Eq. (6). For schematic spectra one finds explicitly an exponential decrease of $\kappa_{0}$ with decreasing spacing of the crossings. For IH the crossings are unstable-stableunstable and generically provide two swaps. However, they can merge to a single swap if the spacings between the crossings are too small, i.e. the triple crossing acts as an effective single one. The dynamical formation of the swaps in the triple-crossed case can be very different depending on the spectrum. The location and widths of the swaps are fixed dynamically by coupled-pendulum solutions together with the corresponding adiabatic invariants.

Multi-angle effects. - SN neutrinos travel on different trajectories and every mode is described by its direction of motion, in addition to $\omega$. Multi-angle effects can cause kinematical decoherence among angular modes 13] and smear the spectral features. However, in spherically symmetric cases the collective motions seem rather robust against multi-angle effects and a single-angle treatment often seems to capture the main effect [14].

The swap factors from multi-angle simulations of our examples are shown as dashed lines in Figs. 2 and 3 The right split in Fig. 3 is robust whereas the left one smears out as previously noted [20, 21]. In Fig. 2 we find significant decoherence across the spectrum. The IH case requires a large number of modes to reach apparent convergence (we used 200 frequency and 15000 angular modes). These issues require a dedicated investigation that is beyond the scope of our work. It seems clear, however, that some of the splits survive multi-angle decoherence as sharp spectral features.

Concluding remarks. - Spectral swaps with concomitant splits are generic for dense neutrino fluxes. They can appear in the $\nu$ and $\bar{\nu}$ channels for both mass hierarchies. We have provided a qualitative explanation for the main features of the multi-split phenomenon. Spectral swaps are nucleated by unstable crossings of the initial flavor spectra. The number and locations of observationally relevant splits depends on the properties of the initial spectra and the effective mixing angle, opening the possibility for observing interesting time-dependent features. Notice that the SN flavor spectra strongly differ between the prompt deleptonization burst, the accretion phase, and the cooling phase. Multi-angle effects smear some of the splits, once more opening the possibility for interesting time-dependent features. It seems that the physics of SN neutrino conversion is much richer than it was thought before and further studies may uncover a number of qualitatively new phenomena.

Acknowledgements.-We thank A. Mirizzi and E. Lisi for fruitful discussions and acknowledge partial support by the Deutsche Forschungsgemeinschaft under grant TR-27 "Neutrinos and Beyond" and the Cluster of Excellence "Origin and Structure of the Universe" (BD and GR), by a Max Planck-India Partnergroup Grant (AD) and by the Alexander von Humboldt-Foundation (AS).

A. Dighe, J. Phys. Conf. Ser. 136, 022041 (2008).

[2] J. T. Pantaleone, Phys. Lett. B 287, 128 (1992).

[3] G. Sigl and G. Raffelt, Nucl. Phys. B 406, 423 (1993).

[4] V. A. Kostelecky and S. Samuel, Phys. Rev. D 52, 621 (1995).

[5] S. Pastor, G. G. Raffelt and D. V. Semikoz, Phys. Rev. D 65, 053011 (2002).

[6] Y. Y. Y. Wong, Phys. Rev. D 66, 025015 (2002).

[7] A. B. Balantekin and Y. Pehlivan, J. Phys. G 34, 47 (2007).

[8] S. Pastor and G. Raffelt, Phys. Rev. Lett. 89, 191101 (2002).

[9] R. F. Sawyer, Phys. Rev. D 72, 045003 (2005).

[10] H. Duan, G. M. Fuller and Y. Z. Qian, Phys. Rev. D 74, 123004 (2006).

[11] H. Duan, G. M. Fuller, J. Carlson and Y. Z. Qian, Phys. Rev. D 74, 105014 (2006).

[12] S. Hannestad, G. G. Raffelt, G. Sigl and Y. Y. Y. Wong, Phys. Rev. D 74, 105010 (2006); Erratum ibid. 76, 029901 (2007).

[13] G. G. Raffelt and G. Sigl, Phys. Rev. D 75, 083002 (2007).

[14] A. Esteban-Pretel et al., Phys. Rev. D 76, 125018 (2007).

[15] H. Duan, G. M. Fuller, J. Carlson and Y. Z. Qian, Phys. Rev. D 75, 125005 (2007).

[16] G. G. Raffelt and A. Yu. Smirnov, Phys. Rev. D 76, 081301 (2007); Erratum ibid. 77, 029903 (2008).

[17] G. G. Raffelt and A. Yu. Smirnov, Phys. Rev. D 76, 125008 (2007).

[18] H. Duan, G. M. Fuller and Y. Z. Qian, Phys. Rev. D 76, 085013 (2007).

[19] H. Duan, G. M. Fuller, J. Carlson and Y. Z. Qian, Phys. Rev. Lett. 99, 241802 (2007).

[20] G. L. Fogli, E. Lisi, A. Marrone and A. Mirizzi, JCAP 0712, 010 (2007).

[21] G. L. Fogli et al., Phys. Rev. D 78, 097301 (2008).

[22] H. Duan, G. M. Fuller, J. Carlson and Y. Z. Qian, Phys. Rev. Lett. 100, 021101 (2008).

[23] B. Dasgupta, A. Dighe, A. Mirizzi and G. G. Raffelt, Phys. Rev. D 77, 113007 (2008).

[24] B. Dasgupta and A. Dighe, Phys. Rev. D 77, 113002 (2008).

[25] H. Duan, G. M. Fuller and Y. Z. Qian, Phys. Rev. D 77, 085016 (2008).

[26] B. Dasgupta, A. Dighe and A. Mirizzi, Phys. Rev. Lett. 101, 171801 (2008).

[27] A. Esteban-Pretel et al., Phys. Rev. D 78, 085012 (2008).

[28] B. Dasgupta, A. Dighe, A. Mirizzi and G. G. Raffelt, Phys. Rev. D 78, 033014 (2008) arXiv:0805.3300.

[29] J. Gava and C. Volpe, Phys. Rev. D 78, 083007 (2008).

[30] G. G. Raffelt, Phys. Rev. D 78, 125015 (2008).

[31] B. Dasgupta et al., in preparation (2009).

[32] G. G. Raffelt et al., in: 4th Workshop on Neutrino Oscillations and their Origin, ed. by Y. Suzuki et al. (World Scientific, Singapore, 2004) astro-ph/0303226.

[33] Animations corresponding to our figures are provided at http://www.mppmu.mpg.de/supernova/multisplits 